\title{
Outsourcing Project Management Services in the Public Sector
}

\author{
A.Vagelatos, H. Tsaknakis, F. Foskolos, T. \\ Komninos RACTI, Greece
}

\begin{abstract}
The increasing demand for e-Goverment services has created considerable challenges for public administration, not only in terms of expertise and experience that are required in order to run development projects, but also from organizational, cultural and legal perspectives. In fact, the ongoing modernization of public administration functions is realized through a large number of complex and technology intensive projects. Most of them are cofinanced by the European Union within the framework of Community Development Programmes. The need for effective implementation of such projects in compliance with the European Commission (EC) directives and for the seamless integration of their outcomes into the organizational structure, has driven public administration officials to seek alternative means for enhancing the operational effectiveness of departments involved in project implementation and for supporting functions related to the utilization of project results and change management. This paper describes a certain case study on outsourcing practice in the Greek Public Sector.
\end{abstract}

\section{Introduction}

The importance of new technologies for any modern public administration has been well established long time ago and various such technologies have been implemented and are already in operation for a large number of public administrations around the world. The added value is primarily due to the automation of business processes and the streamlining of organizational activities that are possible with the use of Information \& Communication Technologies (ICT). However, beyond its obvious and well established importance nowadays, the use of ICT and the optimal utilization of the ICT resources in public administration has many practical challenges both in terms of human resources needed to run the systems and in terms of maintaining and enhancing the utilization of constantly evolving ICT.

In regard to the Ministry of Education, Lifelong Learning and Religious Affairs of Greece (Greek initials: YPEPTH), the use of new technologies has evolved around two main directions: (a) to facilitate and streamline the administrative processes of its administrative structure consisting of a central national office, the regional offices and the public schools all over the country, providing at the same time the necessary interconnection among them, and (b) to support and enhance the teaching and learning practices in schools through the utilization of ICT and the introduction of novel educational activities made possible by ICT. Along these lines, over the past years a large number of ICT-related projects have been designed and implemented either by YPEPTH itself or by organizations supervised by it and acting on its behalf. As a result, a huge ICT infrastructure has been developed covering the administrative units and schools throughout the country (about 15.000 points), specialized computer systems and applications have been developed to support its complex administrative procedures, and a large corpus of educational software and computerbased educational activities have been introduced in schools supported by large scale teacher training programs (more than 100.000 teachers have already been trained).

One of the major tasks of the ministry of education is to facilitate the transition of schools and the educational system in general into the Information Society. Due to the rapid evolution of new technologies, such a task should be dynamic in nature and requires large and complex initiatives to enhance teaching and learning processes throughout the country, to support novel educational activities and practices through the utilization of ICT in schools and to integrate all relevant activities into the educational system and everyday classroom practice.

During the last eight years, emphasis has also been put by YPEPTH on e-government related issues and on implementing ICT projects whose purpose is the facilitation and support of the citizens involved in the education affairs, giving access to relevant information and services. For example, candidate teachers can now apply electronically for a teaching job in a school through an information system operated by YPEPTH.

In addition, YPEPTH implements a large number of projects that are funded by the Operational Programmes of the Community Support Framework (OPCSF) of the European Commission (EC). Such projects include ICT projects (but not only), most of which are eligible for funding under these EC operational programmes. 
The development and utilization of ICT by YPEPTH on the one hand and the design and implementation of many large-scale and complex projects on the other, have created considerable challenges for YPEPTH and unprecedented needs for the following basic reasons: (i) The organizational structure of YPEPTH is adapted to its core functions that are related to education administration and it is not suitable for running projects or for providing ICT services, (ii) There is no adequate permanent personnel with the ICT project management skills and expertise required, neither is it desirable or feasible to create and maintain such expertise inhouse.

In order to resolve such problems and enhance the performance and cost effectiveness of project related activities, the ministry decided to outsource a number of specific operations related to project implementation, including ICT projects, but also other projects as well. In this paper, we describe one such practice where the ministry enhanced the Special Implementation Authority for Educational Programmes (a department of the ministry specifically created to run projects that are eligible for funding from the Community Framework Support Programmes (2000-2006) and National Strategic Reference Framework (Greek initials: ESPA) 2007-2013) by outsourcing some of its services.

\section{Literature Review}

\subsection{Outsourcing}

Outsourcing (or out-tasking as was named by some organizations) has become a widespread practice the last 30 years. Industry observers expect outsourcing to continue growing [1]. Historically, the soft areas such as custodial services, food services, and landscaping have dominated the outsourced work; almost 50 percent of surveyed organizations identify these areas [2]. Nevertheless, a major part of outsourced services has to do with Information Technology (IT) and Project Management (PM), fields which are within the scope of this paper and will be the focus point of the rest of this paper.

There is a significant number of references in the literature concerning outsourcing worldwide [3]. Most of the papers in the literature deal with IT outsourcing whereas other areas of outsourcing (like project management) are less covered. Information systems (IS) outsourcing has become a rather popular issue since companies like Ford or Kodak began to apply it. Nowadays, and after a long period of debate, IS outsourcing is considered as an acceptable way to deal with certain IS tasks. The main focus of outsourcing is on externalizing those organizational activities which are not directly related to the core competences of the organization [4].

Initially, IS outsourcing consisted of an external vendor providing a single basic service to the customer, exemplified by facilities management arrangements where the vendor assumed operational control over the customer's technology assets, typically a data center [5]. Outsourcing of information systems began to evolve in 1963 when Electronic Data Systems (EDS) signed an agreement with Blue Cross of Pennsylvania for the handling of its data processing activities. This was the first time a large business had turned over its entire data processing department to a third party. However, the real interest in outsourcing occurred during the mid1980s when EDS signed contracts with Continental Airlines, First City Bank and Enron. Later on, outsourcing evolved from the one vendor - one client arrangement where the vendor provides apparently all IS services to its client, to complex arrangements involving multiple vendors and multiple clients.

More recently, the industry has seen the growth of two new areas of IS outsourcing: "Web" and "eBusiness" outsourcing where vendors are contracted to provide web-based applications to enable a firm to enter the e-Business era. The latter practice is usually referred to as Application Service Provision (ASP) [6].

Public administration (PA) also started experimenting with IS outsourcing practices driven by market and political trends [3, 7]. The UK's Compulsory Competitive Tendering (CTT) was probably the first relevant example, introduced during the 80's at the local administration level [8], while the USA's National Performance Review (NPR) employed IS outsourcing during the 90's [9]. Many other countries have followed these initial exemplars and some methodologies such as the "Euromethod" [10] and "Information Services Procurement Library" (which has its roots in Euromethod) [11] have been devised to help with this process.

\subsection{The reasons to Outsource}

Outsourcing is defined as "Having work that was formerly done inside the organization performed by an external organization" [12]. A question that often arises is why firms (or other organizations) choose to outsource some activities and retain others? Among the varieties of answers that can be collected, a rather "politically correct" one is that basically outsourcing can be viewed as a compromise between vertical integration (associated with cumbersome hierarchies and bureaucracy) and reliance on market mechanisms (which of course entails cost and a number of risks) [12]. 
But the outsourcing issue is much more complicated, and a comprehensive set of factors should be taken into account before the actual decision is made. This set includes an organization's business model, size, industry characteristics, culture and external environment (to mention just the most important ones). And, of course, the nature of the business sector (e.g. public, private or not-for-profit) plays a very important role in the equation. In [13] it is claimed that a number of studies agree about the main objective of outsourcing initiative (emphasis is on IS outsourcing) and although most of them are common for both the public and the private sector, there are several notable differences.

The need to cut costs and increase efficiency seems to be the most important reason towards an outsourcing decision. Human resource considerations also play an important role in a twofold sense: downsize, for example, the IT department and gain access to high level professionals. In many cases, concentration on core business is also mentioned as an important reason.

On the other hand, as far as the public sector is concerned, decisions about outsourcing projects are most of the times made by high level public officials who are in charge of implementing governmental policies, such as, for example, cost cutting, overcoming problems with recruiting adequately skilled personnel, etc [8].

\subsection{Project Management outsourcing}

Outsourcing Project Management (PM) tasks is another, slightly different concept. When an organization or a public authority is aware of its weaknesses regarding project management, one solution is to outsource these activities to someone with the appropriate expertise. Outsourcing project management tasks [14] brings certain benefits, but also has its limitations. An experienced PM service provider could enrich project teams in a public organization by bringing along project tools and techniques that have been proven effective in practice and also by infusing a different projectoriented work culture. On the other hand, the effectiveness of outsourcing Project Management functions is highly related to a public organization's administrative structure and processes and their resistance to change. Indeed, public administrations have not yet implemented a PM culture. Introducing PM professionals into this environment requires strong support from the top management, otherwise results will be limited. Furthermore, the expectations should be realistic regarding how much a project could be improved by outsourcing the PM tasks: if the project lacks sponsors' support, or is not well defined, or resources are inadequate, then there is no tool or technique that will save it from problems.

\subsection{PM outsourcing's pros and cons}

If the advantages vs. disadvantages of an outsourcing initiative were to be reviewed in the literature, a great number of publications could be possible identified in general. Nevertheless with the focus on public sector only a few of them are relevant and most of them are concentrating on IT business. Table 1 summarizes the findings [13, 14, 15]:

Table 1. Pros and Cons of outsourcing in the public sector

\begin{tabular}{|l|l|}
\hline Pros & Cons \\
\hline $\begin{array}{l}\text { Avoid problems with } \\
\text { recruiting adequately } \\
\text { skilled staff }\end{array}$ & $\begin{array}{l}\text { Dependence } \\
\text { contractor }\end{array}$ \\
\hline $\begin{array}{l}\text { Acceleration of business } \\
\text { process }\end{array}$ & Possible loss of control \\
\hline $\begin{array}{l}\text { Concentration on core } \\
\text { business }\end{array}$ & $\begin{array}{l}\text { Loss of in-house skills } \\
\text { if a know-how transfer } \\
\text { has be designed) }\end{array}$ \\
\hline $\begin{array}{l}\text { Adoption of best } \\
\text { practices }\end{array}$ & $\begin{array}{l}\text { Controversy between } \\
\text { public sector values and } \\
\text { the cost-benefit approach }\end{array}$ \\
\hline & Confidentiality matters \\
\hline
\end{tabular}

The most interesting point to mention here is that a number of studies on outsourcing [15], stress the distinctive nature of public vs. private organizations: they have different goals that are reflected in their ethics and value. Thus a notable fact is that in most cases, outsourcing initiatives in public organizations is mainly directed to other public organizations or not-for-profit ones [13].

\section{Outsourcing in the Ministry of Education in Greece}

In this section, we describe a specific outsourcing practice of YPEPTH. The outsourcing refers to the functions of the Special Implementation Authority for Educational Programmes (Greek initials: EYE) of YPEPTH starting on November 2007.

\subsection{The need for outsourcing}

Near the end of 2007, it became clear to YPEPTH officials that the performance of the projects under the responsibility of EYE were lagging behind expectations in terms of progress of implementation and absorption of budget and that the deadline for completion of all approved EC projects (end of December 2008) was fast approaching. So, it was critical to take immediate measures in order to enhance the performance of EYE and to speed up the process of implementation of all its projects (total budget of 630 million Euros). 
The concept of project management and all the associated functions like scheduling, budgeting, deadlines, resource allocation, reporting, etc., do not fit well into the organizational structure of a public authority like YPEPTH neither it is its core business, especially for projects involving new technologies that are dynamic in nature. Additionally it does not have the culture and adequate expertise to implement projects. In fact, most of the ministry's professional staff are assigned jobs related to their primary domain expertise (i.e. educational experts), or they are involved in necessary administrative work. However, some of the staff (mostly school teachers dispatched to the ministry) has acquired some project management skills after years of involvement, but they are not permanent in such jobs (not permanent in the sense that there are no official placements with that job description) neither are they enough in number for the workload required. On the other hand, hiring additional permanent staff with the appropriate skills to run projects is not considered feasible due to lengthy and complicated hiring procedures in the public sector.

In regard to ICT expertise, there is a rather small number of employees of YPEPTH with certain ICT expertise who are mostly school teachers with specialty in informatics dispatched to the ministry. Primarily, such persons belong to the department of ICT infrastructure of YPEPTH and they are involved in monitoring the operation of some critical infrastructure of the ministry. A smaller number of them are assigned to other departments of the ministry including the Special Programmes Directorate specifically created to run projects (see below). However, there is no established systematic way of maintaining in house expertise on ICT products and services. So, the ministry needs very often external specialized ICT experts to solve its everyday problems.

For all the above reasons, outsourcing some activities related to project implementation has been considered by YPEPTH as absolutely necessary. The Research Academic Computer Technology Institute (RACTI) has been selected by the ministry officials to perform such functions. RACTI has the legal credential to be assigned such a task as a private institution (not-for-profit private legal entity) supervised by YPEPTH, while at the same time it has extensive expertise on PM issues. For this purpose, it was assigned some of the tasks of EYE, including the overall management of the directorate. Within this framework, RACTI created the Ministry of Education Support Office and staffed this office with a group of experienced personnel to carry out the job of supporting the functions of EYE. It was not the first time that such work assignments of YPEPTH to RACTI took place. In fact, for many years RACTI has been a technical consultant of YPEPTH undertaking the implementation of a large number of specific ICT projects on behalf and for the benefit of the ministry. However, such cooperation was basically on a yearly contract basis. This time, the ministry essentially created a form of strategic partnership with RACTI and delegated to it some of its non core functions related to project implementation thus including a whole team of experts into its the organizational structure.

\subsection{Implementation and challenges}

Special Implementation Authority for Educational Programmes (EYE) of the Greek ministry of education had been created in 2002 for the purpose of designing, monitoring, organizing, coordinating, supporting and evaluating projects funded by Operational Programmes of the Community Support Framework and which are of interest to the ministry. For every project that EYE undertakes on behalf of the ministry, it has the responsibility to run all necessary operations for its implementation, from start to completion. It does so in close cooperation with the appropriate (for each project) department(s) of the ministry or the appropriate supervised (by the ministry) organization(s) that are the stakeholders and responsible for the utilization of the deliverables and/or the operation of the project. Furthermore, EYE is subject to audits performed by designated authorities during or after the end of each project.

Some indicative examples of projects implemented or under implementation are the following:

- ICT infrastructures: Computer labs in schools and networking of educational and administrative units.

- Teacher training in basic ICT skills as well as in domain specific ICT-based skills.

- Educational software and ICT-based educational activities for all school disciplines.

- Implementation of the Digital Classroom (the evolution of today's classroom with extensive utilization of ICT).

- Implementation of a Data Center (central data and information repository) and the appropriate applications for the administrative processes of YPEPTH.

- Infrastructure for e-Goverment services that YPEPTH offers to teachers, students, parents and citizens.

- Digitization and archiving of books of the Public Libraries of Greece.

- Procurement and installation of ICT and special equipment for students with special needs.

The first task of RACTI's team was the overall assessment of the situation of each distinct project undertaken by EYE on behalf of YPEPTH. It is pointed out that at that time there were 45 projects with a total budget of 630 million Euros and that the percentage of implementation (in regards to budget 
absorption) was quite low at less than $10 \%$ at the time the support office started its operation near the end of 2007. Furthermore, the schedules for all projects were very tight due to the fact that the official programmatic period was about to end in December 2008 and all EU funded projects should have been completed by that time.

Considering the work that had to be done for a successful completion of the projects, the human resource requirements were estimated per role and per project and a review of the available personnel at YPEPTH was conducted in terms of skills in project management and ICT. Based on this information, it was possible to some extent to rationalize the distribution of workload on people and make the right assignments of jobs to individuals, wherever that was legally and administratively possible.

The work of the members of the RACTI team can be categorized as follows:

- Codification and rationalization of administrative and technical procedures involved in the implementation of projects. Redesign and/or fine tuning of some projects.

- Definition and creation of a monitoring \& Support scheme during the entire lifecycle of all projects concerning legal, financial, technical and administrative issues.

- Task management. Streamlining of tasks to the units of EYE and scheduling of activities. Specific task lists on a daily basis, assignments of tasks to persons.

- Communication management: Communication with appropriate authorities and stakeholders.

- Technical processing of calls for tender for consistency, soundness and compliance to rules and regulations.

- Resource management, including human resources management.

- Direct contribution to certain important projects of EYE.

Each project has its own complexities and peculiarities, schedule, budget and rules of eligibility for funding under the Community Support Framework Programme. Some projects are interconnected in the sense that the intermediate or final results of some are prerequisites and/or should provide input to others. All projects have to follow strict rules and procedures throughout their implementation dictated both by the specific eligibility requirements for funding, as set by the EC, and by the general national procurement rules that should be applied for the assignment of all public projects to outside contractors. Adherence to the rules and procedures is a prerequisite for the eligibility for funding and is subject to multiple layers of controls and audits by the supervising authorities (both national and EC authorities) even after the end of a project and for a period of 5 years afterwards. Deviations from the rules at any time and for any action related to a project may render the entire project ineligible for funding by the EC and may result in financial damages for the ministry. In order to avoid such eventualities and enforce full compliance to the law, the ministry (like all public organizations implementing projects) has set up internal procedures requiring, among other things, that any decision taken in the course of the implementation of a project should pass through the regular chain of command of the ministry and be duly signed and dated by the appropriate officials each time.

On the other hand, there is a great variety of projects all of which are subject to the same rules and regulations. All projects have to be coordinated by the same pool of people, primarily by the staff of EYE. Due to human resource constraints it is neither feasible nor would it be practical and cost effective to have distinct project teams for each project. So, there must be overlaps and the same person may be involved in several project teams and in different positions in them according to his/her skills and the special needs of each project.

One major challenge for RACTI's team was the effective combination of two different requirements: (i) enforcement of efficiency, speed, deadlines and results in the work environment of a public organization, and (ii) full compliance with rules, regulations as well as with the internal procedures of YPEPTH in all phases of all projects under implementation. In order to achieve a workable combination of these factors, the team had also taken into consideration that for all actions within the framework of the implementation of projects, the staff of EYE and the ministry officials were legally liable in the end, for any mistakes or omissions. So, all actions were carefully designed so as to avoid such situations while enforcing efficiency and speed.

The team utilized its expertise and experience in project management as well as its work culture to establish a working relationship and mutual trust with the people of EYE. A deep understanding of the work culture, the procedures, the rules and the existing administrative structure of YPEPTH and EYE was necessary so as to properly align and fine tune the initiatives of the team with the mechanism of EYE. A lot of effort on the part of the team had to do with finding appropriate trade-offs among the requirements for efficiency in project implementation, the rules and procedures that had to be followed at every step, the work culture of the people and the administrative structure. Cooperation, team spirit, smooth adaptation, continuity, efficiency, strict adherence to the rules and working within the system, were basic principles that were followed throughout this endeavor. As a result, an effective mechanism was created and is currently operational, that balances such factors 
sufficiently well and delivers projects on time and within budget.

\section{Discussion}

Outsourcing project management tasks appears to be necessary and beneficial for a public authority when complex and demanding development projects have to be implemented. The main reason is that in most cases, implementation of such projects is not a core business of a public organization; hence, its organizational structure and work environment are usually not appropriate for such endeavors. On the other hand, more often than not, there is a considerable resistance to change in a public organization. However, change and adaptation are key words for any such project and, also, the main objective and prerequisite in most cases.

In regard to the work presented here, one of the significant benefits of YPEPTH from outsourcing to RACTI certain project management tasks, was that RACTI's team, apart from its expertise, brought along certain project management tools and techniques that helped improve overall projects' results. This way, a know-how transfer to YPEPTH's staff regarding PM tools and techniques has taken place and continues on. On the other hand, the interaction among staff members of both organizations (that is absolutely necessary for any project and at any phase of its lifecycle), resulted in an infusion of certain work habits and culture of RACTI's staff (that is similar to the work culture of the private sector organizations) to YPEPTH's colleagues. It also resulted in the creation of a team spirit to the mutual benefit of both organizations. Such a cooperation and work environment was to a certain extent necessary due to the requirements of the projects and the overall work that had to be done in a narrow time frame.

Soon after the undertaking of the project management functions on behalf of the YPEPTH, it was realized that one of the most significant difficulties hindering effective project implementation was due to the very organizational structure of the agency (EYE) of the ministry which is authorized to do exactly this job, i.e. to run projects. In fact, its organizational structure was no different than any other public agency's organizational structure tailored to do routine administrative work and having as a main priority (or only priority in some cases) the adherence to the regulatory and legal directives. The latter are of course necessary, but they are hardly enough when it comes to running rather innovative projects requiring flexibility, creativity, proactiveness, speed, efficiency and high performance under (usually) tight constraints on time, budget and human resources.
However, such an organizational structure is mandated by law and cannot be changed for any reason. So, one of the challenges was to find a way to infuse a "project oriented" culture and to provide the means, the motivation and the direction toward more efficiency without violating any of the legal and regulatory restrictions. After some initial discussions, the decided strategy was to introduce standardization on the routine processes; indeed, such standardization was applied and resulted in significant savings in time and human resources. Additionally, another action towards this direction was to enforce reporting and progress reviewing on a regular basis for each particular task and each particular project.

Along these lines, an ISO-like standard methodology has been introduced and became an integral part of the procedures of EYE. This methodology includes standard procedures in handling data and document flows for the entire agency, document templates, standard forms and an IT-based system supporting the entire work flow process. All members of EYE are now using this documents and work flow management tool after a period of on-the-job training.

\section{Issues for future consideration}

During the period of involvement in the outsourcing project discussed here (which continues on at the time of writing this paper), it became clear to the project team that a number of new problems and challenges (compared to our previous experiences) have to be dealt with on a continuous basis and new lessons have to be learned along the way. The most significant ones that are currently under consideration are outlined below.

In the Greek Public sector a major drawback is that classical metrics and concepts used in project management, such as man-hours, time sheets, internal cost, and task assignment \& scheduling, performance measures, etc. are not being used by any (with a few exceptions) public organization. Therefore, it is not easy to measure cost versus benefit accurately. Only gross estimates can be made based on the total money and time spent for the projects as well as the number of people involved in the implementation of the projects. In addition to that, there is no mechanism in place to measure the impact of the results of a project on the educational community. However, this is far from being satisfactory: it is necessary to define appropriate key performance indicators to measure the impact on the educational community of the results of a project and specialized work has to be done in this direction. Additionally, a mechanism should be deployed to measure cost of resources and performance of individuals and teams. Such a mechanism would enable a cost versus benefit analysis for each project. 
Another issue is that there is a significant communication gap between the stakeholders: a) the state personnel, b) RACTI team members and, c) the private sector professionals (contractors, etc). The nature of this problem is that the first group does not fully realize that there is more than one way of doing things within the framework of the law, whereas the latter group does not often realize that there are strict rules mandated by law that should always be followed in the public sector. This problem can be alleviated only if both parties are willing to cooperate for their mutual benefit and move one step ahead at a time. In fact, one must try to understand the way the other think, to step into his shoes, and discover the way the other walk. There is more than one path to follow, but there are paths and not free space. It turns out that it takes quite some time and effort from all sides to understand this and even more time to achieve a desirable level of communication and interaction. There is a need for some sort of cultural interaction. Such interaction should involve concessions from all sides and even changing certain pre-existing work habits and modes of operation in order to make progress and have tangible results.

In terms of technology and knowledge transfer from the outsourcing partner ( RACTI) to the state employees, the experience gained seems to indicate that this is generally quite difficult to accomplish when both parties are working under tight schedules and are under pressure to fulfill the tasks they are assigned. This transfer becomes possible mainly during time periods where the job pressure is relatively low. That is also the appropriate time for long term planning, both strategic and operational, for offline paperwork like creating templates and forms, for reviewing and updating the needed infrastructure like computer systems, information systems and tools, collaboration platforms, project management tools. Another aspect of knowledge transfer refers to establishing standards throughout the agency, creating standard documents and procedures for monitoring, evaluation and staff training, which also should be done during low work pressure periods.

Another major PM phase which was realized that is missing in public sector is project evaluation. It is important for the state to realize that the appropriate time and resources should be reserved to look back and try to perform a trustworthy evaluation of all the tasks and all the work that has been made. Such expost evaluation should be performed thoroughly by examining all key factors of any project, in terms of quantity as well as quality. For example, for every project undertaken, one should try to answer questions such as: what was the average man-month cost for the administrative work relative to the total project cost? How does this compare to other similar projects? Are there any special factors that affect the progress of a project? What are the key points that need to be in our check list for future reference? Have we identified all the problems? And based on the outcomes of this evaluation, certain measures should be taken in order to improve the operational effectiveness of EYE.

\section{Conclusions}

As far as results are concerned, during a period of a little more than two years (Nov. 2007 - Dec. 2009), there was an impressive improvement of the effectiveness of the directorate in terms of rates of progress in project implementation. In fact, in terms of budget absorption which is an easily measurable performance index, although it does not fully reflect the implementation project, the percentage was about $10 \%$ at the end of 2007 and increased to $80 \%$ by December 2008. In addition to the above, all audits that have been performed so far by the designated authorities for a variety of projects have been successful and no deviations from legal rules and procedures were detected whatsoever.

Finally, it should be pointed out that the overall outsourcing experience described here has given rise to a new mentality and know-how toward organizing the implementation and integration of large and complex projects into a public organization. It was the source of valuable experience for the creation of rules, procedures and methodologies that can potentially help the ministry of education handle its future challenges.

\section{References}

[1] Levit, N. (2007) 'The Changing World of Outsourcing', IEEE Computer, 40(12), pp. 13-16.

[2] Cleland, D. and Ireland, L. (2002) Project Management, McGraw-Hill.

[3] Marco-Simó, J., Macau-Nadal, R. and Pastor-Collado, J. (2007) 'Information systems outsourcing in public administration: an emergent research topic', in Proceedings of European and Mediterranean Conference on Information Systems 2007 (EMCIS2007), June 24-26 2007, Valencia, Spain

[4] Lee, J., Huynh, M., Kwok, R. and Pi, S. (2003) 'IT outsourcing evolution--past, present, and future'. Communications of the ACM, 46(5), pp. 84-89.

[5] Dibbern, J., Goles, T., Hirschheim, R. and Jayatilaka, B. (2004) 'Outsourcing: A Survey and Analysis of the Literature', The DATA BASE for Advances in Information Systems, 35(4) Fall 2004.

[6] Kern, T., Lacity, M. C. and Willcocks, L. (2002) Net Sourcing: Renting Business Applications and Services Over a Network, Upper Saddle River, NJ: Prentice-Hall. 
[7] Sarivougioukas, J. and Vagelatos, A. (2002) 'IT outsourcing in the Healthcare sector: The case of a state general hospital', in Proceedings of SIGCPR Conference, Kristiansand, Norway, May 2002.

[8] Hancox, M. and Hackney, R. (1999) 'Information technology outsourcing: Conceptualizing practice in the public and private sector', in Proceedings of the 32nd Annual, International Conference on System Sciences, Hawaii, USA.

[9] Beyah, G. and Gallivan, M. (2001) 'Knowledge management as a framework for understanding public sector outsourcing", in Proceedings of the 34th International Conference on System Sciences, Hawaii, USA.

[10] "Euromethod", http://projekte.fast.de/Euromethod/ index.htm (22 January 2010)

[11] "Information Services Procurement Library", http://projekte.fast.de/ISPL/ (22 January 2010)

[12] Beaumont, N. and Khan, Z. (2005) 'A Taxonomy of Refereed Outsourcing Literature', working paper, Monash University Business and Economics, http://www.buseco.monash.edu.au/mgt/research/workingpapers/2005/wp22-05.pdf (22 January 2010).

[13] Jedd, M. (2004) 'Outside In', PM Network, 18 (1), pp 46-50.

[14] Rodriguez, A. (2007) 'Trends in Argentina: Outsourcing Project Management'. PM World Today, Vol. IX, Issue VII.

[15] Yu, T.K. and Cheng, F. (2007) 'Information System Outsourcing in Government: Public and Private Sector long-term Alignment', in Proceedings of International Conference on Business and Information, July 2007, Tokyo, Japan. 